DOI: $10.35643 /$ Info.26.2.10

Artículo original

\title{
Evaluación de los planes de estudios de la Facultad de Información y Comunicación: el rol de los docentes en la implementación de propuestas formativas con enfoques pedagógicos flexibles
}

\author{
Evaluation of the Faculty of Information and \\ Communication curricula: the role of teachers in the \\ implementation of training proposals with flexible \\ pedagogical approaches
}

Avaliação dos currículos da Faculdade de Informação e Comunicação: o papel dos professores na implementação de propostas de formação com abordagens pedagógicas flexíveis

Javier Canzani ${ }^{1}$ ORCID: 0000-0001-7253-9628

Natalia H. Correa² ORCID: 0000-0001-6994-0314

Fabián Hernández ${ }^{3}$ ORCID: 0000-0003-2173-3417

Varenka Parentelli ${ }^{4}$ ORCID: 0000-0003-2033-7949

Ema Zaffaroni ${ }^{5}$ ORCID: 0000-0002-7363-5651

${ }^{1}$ Universidad de la República, Facultad de Información y Comunicación,

Departamento Fuentes Documentales, Recursos y Servicios de Información. San Salvador 1944, Montevideo, Uruguay. Correo electrónico:

javier.canzani@fic.edu.uy.

${ }^{2}$ Universidad de la República, Facultad de Información y Comunicación, Unidad de Apoyo a la Enseñanza. San Salvador 1944, Montevideo, Uruguay. Correo electrónico: natalia.correa@fic.edu.uy.

${ }^{3}$ Universidad de la República, Facultad de Información y Comunicación,

Departamento Fuentes Documentales, Recursos y Servicios de Información. San Salvador 1944, Montevideo, Uruguay. Correo electrónico:

fabian.hernandez@fic.edu.uy. 
${ }^{4}$ Universidad de la República, Facultad de Información y Comunicación, Unidad de Apoyo a la Enseñanza, San Salvador 1944, Montevideo, Uruguay. Correo electrónico: varenka.parentelli@ @ic.edu.uy.

${ }^{5}$ Universidad de la República, Facultad de Información y Comunicación, Departamento de Ciencias Humanas y Sociales, Historia Contemporánea. San Salvador 1944, Montevideo, Uruguay. Correo electrónico: ema.zaffaroni@fic.edu.uy

\title{
Resumen
}

Desde el año 2012, las carreras universitarias de la Facultad de Información y Comunicación (Archivología, Bibliotecología y Comunicación) implementan planes de estudio diseñados según lo establecido por las disposiciones de la Ordenanza de estudios de grado y otros programas de educación terciaria del 2011. La articulación y la flexibilidad son dos de los principios orientadores que rigen las propuestas formativas de las carreras. Los objetivos del proyecto estuvieron dirigidos a optimizar la implementación de los planes de estudio y mejorar la calidad de la enseñanza universitaria. Se propuso la realización de instancias de intercambio con docentes para potenciar el conocimiento de los planes de estudio, discutir fortalezas y debilidades y reflexionar sobre el rol docente en el marco de la flexibilidad curricular. La metodología fue de tipo cualitativa, con entrevistas grupales complementadas con datos cuantitativos de una encuesta y la realización de un conversatorio final. Entre los resultados se destaca un uso indistinto en las conceptualizaciones sobre plan de estudio, malla curricular e implementación. No es utilizado como instrumento en la orientación de los estudiantes. Por último, se advierte el interés y la necesidad de crear espacios para la discusión sobre los planes de estudio y sus implementaciones.

\section{Palabras clave: PLAN DE ESTUDIO; FACULTAD DE INFORMACIÓN Y COMUNICACIÓN; ENSEÑANZA UNIVERSITARIA; ENFOQUE PEDAGÓGICO FLEXIBLE; URUGUAY.}

\begin{abstract}
Since 2012, the university careers of the Faculty of Information and Communication (archives, libraries and communication sciences) have implemented curricula designed in accordance with the provisions of the Graduation Studies Ordinance and other Higher Education Programs of 2011. The study project has aimed to optimize the implementation of the study plans and improving the quality of university teaching. The realization of instances of exchanges with professors to enhance the knowledge of the study plans, discuss strengths and weaknesses, and reflect on the teaching role in the framework of curricular flexibility were proposed. The methodology was of a qualitative type, with group interviews, complemented with quantitative data from a survey, and the holding of a final meeting. Among the results, there is an indistinct use in conceptualizations about the study plan, curriculum, and implementation, which is not used as an instrument in the orientation of students. Finally, there is interest
\end{abstract}


and the need to create spaces for the discussion on the study plans and their implementations.

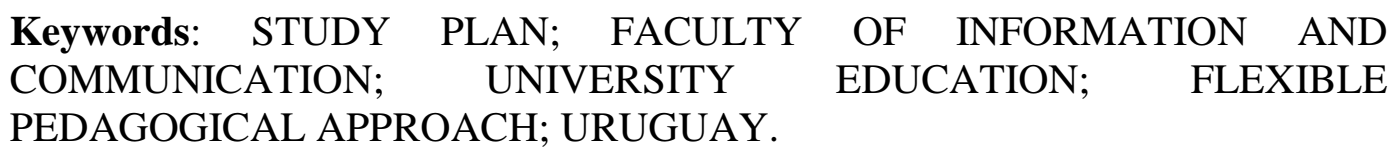

\section{Resumo}

Desde 2012, as carreiras universitárias da Faculdade de Informação e Comunicação (Arquivologia, Biblioteconomia e Comunicação) têm implementado planos de estudos concebidos de acordo com o disposto na Portaria de Graduação e demais Programas de Ensino Superior de 2011. Articulação e flexibilidade são dois dos princípios norteadores que norteiam as propostas formativas das carreiras. Os objetivos do projeto visam otimizar a execução dos planos de estudos e melhorar a qualidade do ensino universitário. Propôs-se realizar intercâmbios com os docentes para aprofundar o conhecimento dos planos de estudos, discutir potencialidades e fragilidades e refletir sobre o papel docente no âmbito da flexibilização curricular. A metodologia foi qualitativa, com entrevistas em grupo, complementada com dados quantitativos de uma pesquisa e uma discussão final. Dentre os resultados, destaca-se um uso indistinto nas conceituações de currículo, malha curricular e implementação. Não é utilizado como instrumento de orientação dos alunos. Por fim, constata-se o interesse e a necessidade de criar espaços de discussão sobre os planos de estudos e suas implementações.

Palavras-chave: PLANO DE ESTUDOS; FACULDADE DE INFORMAÇÃO E COMUNICAÇÃO; ABORDAGEM PEDAGÓGICA FLEXÍVEL; URUGUAI.

Fecha de recibido: 15/07/2021

Fecha de aceptado: 14/09/2021

\section{Introducción}

El presente artículo recoge los resultados de la implementación del proyecto «Evaluación de los planes de estudios de la FIC: el rol de los docentes en la implementación de propuestas formativas con enfoques pedagógicos flexibles». Desde el año 2012, las licenciaturas en Archivología, en Bibliotecología y en Comunicación de la Facultad de Información y Comunicación (FIC) implementan planes de estudio (PE) diseñados según lo establecido en las disposiciones de la Ordenanza de estudios de grado y otros programas de educación terciaria (OG) 
aprobada en octubre del 2011. En este sentido, la articulación y la flexibilidad son dos de los principios orientadores que rigen las propuestas formativas de las mencionadas carreras. Con este enfoque pedagógico, el estudiante es concebido como un sujeto activo en el diseño de su itinerario curricular. Así, en el plan de estudio de la Licenciatura en Comunicación se explicita lo siguiente:

Es desde este contexto que se propone el diseño de un nuevo Plan de Estudios, basado en una concepción pedagógica que atiende el creciente grado de autonomía de cada estudiante a lo largo de su formación. Con este plan se apunta a una mayor flexibilidad, se establecen espacios y actividades que articulan e integran las funciones universitarias de enseñanza, investigación y extensión, y se propende a una real integración entre teoría y práctica, posibilitando un verdadero equilibrio entre la formación profesional y académica [...] Por consiguiente, el diseño curricular responde a dichas políticas, brindando alternativas de formación flexibles que atiendan la diversidad de intereses de cada estudiante (Universidad de la República, 2012b, pp. 4-5).

A su vez, en el plan de estudio de las licenciaturas en Archivología y Bibliotecología se plantea la

tendencia a la flexibilización, en las siguientes dimensiones: a) ampliación de las oportunidades formativas, otorgando mayor autonomía al estudiante para elegir las actividades académicas de su formación; $b$ ) opcionalidad, procurando que la oferta y cursado de unidades curriculares optativas contribuyan a perfilar diferentes orientaciones, asegurando a la vez que los egresados de una misma carrera tengan la formación que requieren las competencias y el perfil profesional; $c$ ) mayor relacionamiento y articulación con otras carreras del Área Social de la Udelar (mediante cursos optativos en común, cursado de electivas, reválidas automáticas, etc.), [...] d) articulación de los intereses estudiantiles con las demandas del entorno social. En síntesis, un proyecto formativo flexible, $[\ldots]$ que permita las movilidades estudiantiles y las transformaciones curriculares que exige la cambiante realidad (Universidad de la República, 2012a, pp. 8-9).

Antes de seguir adelante, es necesario precisar qué entendemos por flexibilidad curricular. Se trata de una estrategia para la promoción de la formación integral y el desarrollo de la creatividad y de la autonomía para la búsqueda de conocimiento (Díaz Villa, 2002). Asimismo, la flexibilidad curricular, además de referirse a la oferta y el rol del estudiante, hace hincapié en la oferta programática.

La flexibilidad de los cursos debe extenderse a la flexibilidad de su oferta. [...] Las unidades académicas de las instituciones [...] pueden o deben propiciar, dentro de su oferta global, cursos integrados que articulen diversos componentes y disciplinas dentro de un campo de formación y que, fundamentalmente, puedan ser objeto de diseño y ejecución por grupos de 
profesores de una o varias unidades académicas. Esto puede incentivar la utilización de estrategias pedagógicas socializadas, participativas y flexibles que tiendan a superar el aprendizaje pasivo (Díaz Villa, 2002, pp. 84-85).

En este marco, con enfoques pedagógicos flexibles, los PE de las carreras de la FIC establecen espacios que integran las tres funciones universitarias, centran la atención en la figura del estudiante como protagonista de su formación, con lo cual se propende al desarrollo de su autonomía. Para cumplir con lo anteriormente expresado, se ofrecen trayectorias flexibles, diferentes orientaciones y la necesidad de transitar por espacios curriculares de otras carreras y servicios para completar su formación de grado.

No obstante, es importante considerar lo que propone Ezcurra (2007) en relación con la diferencia que establece entre el alumno esperado y el alumno real. La autora relaciona al primero con las expectativas que los $\mathrm{PE}$ tienen al momento de ser diseñados, es decir, que sea autónomo y capaz de liderar su propio proceso. No obstante, los estudiantes que transitan por la carrera y que Ezcurra llama reales, no coinciden necesariamente con los esperados.

Si bien en la FIC ya se han desarrollado estudios con el objetivo de evaluar la implementación de los PE, este trabajo tiene como valor diferencial sobre los anteriores el abordaje de la evaluación desde la perspectiva de la mirada de los docentes. En este sentido, toda la información relevada, a través del diseño metodológico y el uso de instrumentos a tales efectos, fue obtenida a partir de la experiencia y percepción de los docentes desde sus roles como actores institucionales.

\subsection{Antecedentes}

Como ya se ha hecho referencia, a lo largo de los años de implementación de los PE, se han llevado adelante varias acciones para su evaluación. A continuación, se listan sintéticamente:

a) Años 2013-2019: La Unidad de Apoyo a la Enseñanza (UAE), junto con la docente de Apoyo a la Investigación, realizaron un estudio longitudinal y comparativo de la primera y segunda generación del PE de la Licenciatura en Comunicación. A través de encuestas y grupos focales de estudiantes, 
además del análisis de escolaridades, se identificaron motivos y expectativas al ingreso a la carrera, obstáculos y nivel de dificultad para el tránsito y avances y criterios para la toma de decisiones de los recorridos curriculares en relación con la orientación profesional elegida.

b) Año 2016: Integrantes de las comisiones de carrera (CC) de las licenciaturas en Archivología y en Bibliotecología, junto con la UAE, implementaron un proyecto, aprobado y financiado por la Comisión Sectorial de Enseñanza (CSE), para la evaluación del PE a través de una reunión con los directores de departamento, reuniones con docentes, grupos focales con estudiantes y análisis de sus escolaridades. Se elaboró un documento con resultados preliminares que se utilizó en una jornada interórdenes. Se redactó un informe final que fue tomado como insumo para trazar líneas de acción para la optimización de la implementación del PE.

c) Año 2016: La CC de la Licenciatura en Comunicación, junto con la UAE, realizaron una evaluación colectiva, con intercambio entre docentes y análisis de escolaridades. Se elaboró una propuesta de adecuación de la oferta curricular a los efectos de optimizar el tránsito de los estudiantes, específicamente, en la elección de la orientación en su trayectoria formativa. También se propuso fortalecer la formación en el uso de la lengua.

d) Años 2017-2020: La UAE realizó un estudio longitudinal de la generación 2017 para analizar y evaluar la implementación del PE 2012 de las licenciaturas en Archivología y en Bibliotecología. A través de la realización de encuestas, grupos focales y análisis de escolaridades, se analizó el nivel de avance de los estudiantes y se identificaron los espacios de referencia que tienen para informarse ante la toma de decisiones respecto de su tránsito por las carreras. Se indagó en el nivel de satisfacción de los estudiantes en relación con las expectativas con las cuales ingresaron a la carrera y se indagó en el cumplimiento de los requisitos del PE para el egreso, en el trayecto final, y sus proyecciones en relación al término de la carrera. 
e) Año 2019: El claustro de la FIC, junto con las coordinaciones de carrera y la UAE, organizaron una jornada de intercambio interórdenes, en donde se abordó el enfoque conceptual pedagógico de los PE. Se realizó una exposición conceptual sobre el rol docente y se analizaron las modificaciones implementadas en las mallas curriculares de las tres carreras.

\subsection{Justificación}

Dada la complejidad de la implementación de PE con enfoques pedagógicos flexibles, es relevante dar continuidad a la evaluación sistemática, a fin de identificar aspectos a mejorar para el avance de los estudiantes y, en consecuencia, el cumplimiento de los requisitos para el egreso.

En relación con lo anterior, si bien existen varios estudios que reúnen insumos relevantes para ello, se entiende necesario continuar identificando dificultades y obstáculos en el tránsito de los estudiantes y, sobre todo, en el egreso. Según la consulta realizada para la redacción de este artículo, la cantidad de egresos de los PE 2012 de la FIC a diciembre del año 2020 fue el siguiente:

- Licenciatura en Archivología: 8;

- Licenciatura en Bibliotecología: 14;

- Licenciatura en Comunicación: 230.

Considerando los ingresos desde el año 2013 (año de inicio de implementación de los PE [1] en Montevideo) hasta el año 2016 [2], se calcula el siguiente promedio por carrera:

- Licenciatura en Archivología: 67;

- Licenciatura en Bibliotecología: 89;

- Licenciatura en Comunicación: 811.

Es de hacer notar que los datos que se presentan son aproximaciones, en tanto hay ingresos considerados que refieren a estudiantes de planes anteriores que se han cambiado al plan 2012. No obstante, esta cifra no es significativa. 


\section{Objetivos del proyecto}

El objetivo general del proyecto era optimizar la implementación de los PE de las carreras de la FIC, con el fin último de mejorar la calidad de la enseñanza universitaria.

Para ello, se propusieron como objetivos específicos:

a) Promover en los docentes la apropiación de la propuesta formativa de las carreras de la FIC. Identificar fortalezas, debilidades, oportunidades y obstáculos de la implementación de los PE.

b) Reflexionar sobre el rol que presentan los docentes en la implementación de $\mathrm{PE}$ a partir de enfoques pedagógicos flexibles.

c) Reunir insumos para el trazado de estrategias y acciones en relación con la implementación de los PE, con el propósito de promover el avance de los estudiantes en las carreras de grado de la facultad.

\section{Metodología}

La metodología fue, principalmente, de corte cualitativo, consistente en entrevistas grupales y en un conversatorio final, aunque se complementó con datos cuantitativos a partir de la implementación de una encuesta.

\subsection{Técnicas}

Se emplearon las siguientes técnicas para la recolección de información.

- Encuesta autoadministrada: Con el propósito de realizar una primera aproximación en torno a las categorías de análisis y reunir información para el diseño de la pauta para las reuniones con los departamentos, se implementó una encuesta autoadministrada a través de un formulario Google.

- Entrevistas grupales: A los efectos de profundizar en los aspectos relevados, se construyó una pauta de intercambio que fue implementada 
en las reuniones con los integrantes de los departamentos académicos de la FIC.

- Conversatorio: Como cierre para el relevamiento de información, se implementó un espacio de intercambio con foco en algunas preguntas dinamizadoras.

\subsection{Categorías de análisis}

La información relevada a través de las distintas técnicas se organizó con base en las siguientes categorías de análisis:

- autopercepción docente sobre nivel de conocimiento del PE, de la malla curricular y de su implementación;

- autopercepción docente sobre la capacidad de dar respuesta a las consultas de los estudiantes;

- percepción sobre las trayectorias y avances de los estudiantes;

- acciones desarrolladas por los docentes para la promoción y avance del tránsito curricular de los estudiantes;

- cómo se informan los docentes sobre los cambios que se realizan en la implementación de los PE (malla curricular, disposiciones, etcétera).

\section{Resultados}

A continuación, se comparten los resultados obtenidos a partir de la implementación de los distintos instrumentos. 


\subsection{Encuesta}

\section{a) Datos de contexto}

En el período mayo-junio del año 2020, se aplicó la encuesta autoadministrada y se recibieron 65 respuestas de un total de 191 docentes de los departamentos de la FIC (34\%). Los más representados en los resultados de la encuesta fueron los del Instituto de Información y, del total de los docentes que respondieron la encuesta, fueron mayoría aquellos que tienen entre 16 y 20 horas semanales en sus cargos, con un 38,4\%. El 63,1 \% de los docentes que respondieron tiene ocho o más años de experiencia docente en la carrera, por lo que ya estaban dictando clase cuando se implementaron los nuevos planes de estudio. Es importante tener en cuenta que, aunque la muestra no es representativa, marca una tendencia del comportamiento de los docentes en cuanto a su relación con la temática abordada por este proyecto.

\section{b) Conocimiento sobre el pe y su implementación.}

El 95,4\% de los docentes que respondieron la encuesta dice conocer el PE y, de ellos, la gran mayoría $(75,4 \%)$ leyó el correspondiente a su carrera el año 2019 o el 2020. El 92,3\% de los docentes encuestados afirma conocer los requisitos del plan para el egreso. Al consultarlos sobre el conocimiento de las disposiciones de la implementación del PE, el 75,4\% afirma conocerlas y, de ellos, el 91,8\% expresa estar al tanto de las actualizaciones. Asimismo, casi todos los docentes encuestados $(95,4 \%)$ dicen estar al tanto de las actualizaciones de la malla curricular de su carrera. La gran mayoría de los docentes encuestados $(92,2 \%)$ manifiesta tener en cuenta el PE al momento de elaborar el programa de su unidad curricular (UC). El total de los docentes que respondieron la encuesta se divide casi a la mitad entre quienes dicen estar al tanto de los egresos y avances estudiantiles y quienes no lo están.

\section{c) Capacidad para la orientación de estudiantes.}

La gran mayoría de los docentes siente que no siempre están preparados para orientar a los estudiantes cuando estos preguntan sobre algún aspecto relacionado con el PE. Al cruzar información sobre los años de experiencia docente, tomando 
aquellos docentes que están desde el inicio de la implementación de los planes de estudio (ocho años o más, 63,1 \% de los docentes encuestados), el porcentaje de quienes dicen sentirse siempre en condiciones de brindar información a los estudiantes es del 15,4\%. De ellos, el $70 \%$ son docentes de las licenciaturas en Archivología y en Bibliotecología y el $30 \%$ de la Licenciatura en Comunicación. El 44,6\% dice «a veces», a excepción de un docente, quien manifiesta que «nunca».

\subsection{Reuniones con los departamentos}

Se llevaron a cabo nueve reuniones (una por cada departamento), con la participación de un total de 72 docentes, que constituye aproximadamente el $38 \%$ del total de los docentes de los institutos y del Departamento de Metodología [3]. El departamento con mayor participación fue el de Tratamiento y Transferencia de la Información, con la presencia de 17 docentes.

A continuación, se presenta un esquema de los resultados obtenidos a raíz de los aspectos planteados:

\section{a) Autopercepción sobre el nivel de conocimiento.}

- Se observa una confusión conceptual entre PE y malla curricular $-\mathrm{y}$ su implementación-, que algunos docentes mencionan que podría provenir del cambio de filosofía respecto de los planes anteriores.

- Se percibe un conocimiento general de los PE, aunque no de aspectos particulares.

- Existe un conocimiento de la porción de la malla curricular que corresponde al departamento en el que trabaja el docente (en la carrera de Comunicación se traduce en la orientación curricular en la que se encuentra el docente) y escaso conocimiento del resto.

- En la implementación se manifiesta una dificultad en la oferta de actividades académicas que permitan a los estudiantes cumplir con los requisitos de egreso: investigación, extensión, prácticas preprofesionales, electivas. 


\section{b) Autopercepción sobre la capacidad de dar respuesta a los estudiantes}

- Los docentes que se encuentran en determinados espacios de toma de decisiones (por ejemplo, integrantes de comisiones de carrera, participantes en el diseño de los $\mathrm{PE}$ ) se autoperciben con un mayor conocimiento para brindar respuestas a consultas de los estudiantes.

- Se considera que el papel del coordinador del equipo (departamento, sección académica) es fundamental para que el resto de los docentes estén informados y se sientan en condiciones de responder dudas.

- Se reitera que la mayor seguridad para apoyar a los estudiantes en sus consultas se produce cuando estas se presentan en el ámbito de actuación o temática de especialización del docente.

\section{c) Sobre las trayectorias y avances de los estudiantes.}

- La percepción de los docentes es que la principal motivación de los estudiantes para la construcción de sus itinerarios curriculares es obtener los créditos necesarios para avanzar en su carrera o egresar.

- Respecto de las optativas y la elección del orientador del trabajo final de grado (TFG), la visión es que los estudiantes no se encuentran en condiciones de tomar decisiones totalmente fundamentadas en lo estrictamente disciplinario y que, frecuentemente, se opta por afinidad personal con un docente particular o bien con un equipo docente.

- En cuanto a los obstáculos identificados en las trayectorias de los estudiantes, los docentes perciben:

- Un primer factor en el TFG que dilata el egreso. En Archivología y en Bibliotecología se trata de un fenómeno histórico (una de cuyas razones puede encontrarse en el acceso temprano al mercado laboral que impacta en la dedicación a la finalización de la carrera). En Comunicación se percibe como un obstáculo por ser una incorporación del actual $\mathrm{PE}$ y también por el escaso número de docentes para las tutorías en relación con el número de estudiantes. 
- Un segundo factor que se encuentra, como ya se mencionó, en las posibilidades de oferta de actividades tendientes a cumplir con los requisitos de los PE (investigación, extensión, prácticas preprofesionales, electivas [4]).

O Un tercer factor en los horarios de dictado de ciertas UC de Archivología y Bibliotecología. Por un lado, superposición entre optativas y obligatorias y, por otro, UC que se dictan en un solo turno.

\section{d) Acciones docentes para la promoción del tránsito curricular de}

\section{los estudiantes}

- Algunos docentes responsables de UC mencionaron que no se realizaron modificaciones sustantivas a los programas, pues estos ya se ajustaban a los principios pedagógicos orientadores del $\mathrm{PE}$.

- En las UC en las que se debió realizar ajustes, se plantea que esto llevó un esfuerzo importante, pues implicó:

- crear una orientación curricular completa desde su inicio;

- cambiar el enfoque del curso para contemplar la filosofía de los nuevos planes;

○ trasladar contenidos del ciclo inicial a ciclos más avanzados en las carreras, entre otros, creando nuevas optativas, talleres o seminarios;

○ modificar las formas de evaluación.

- En cuanto a los desafíos de los cambios en los cursos, estos estuvieron:

○ en la semestralización de unidades curriculares anuales;

- en la complementariedad entre rol profesional y aporte al campo académico;

○ en la vinculación teoría-práctica. 
- Se menciona que la actualización de los contenidos no siempre se produce estrictamente por los cambios de planes, sino también de acuerdo a los avances del campo disciplinario.

- Finalmente, se resaltan las siguientes acciones ejecutadas por los equipos docentes para promover el cumplimiento de los requisitos propuestos por los PE:

- incorporación de estudiantes a las líneas de investigación y de extensión existentes;

○ implementación de Espacios de Formación Integral (EFI);

○ desarrollo de espacios de prácticas preprofesionales;

- propuestas de créditos incrementales en el marco de las unidades curriculares, particularmente para el cumplimiento de créditos de investigación;

- estímulo a la presentación de proyectos en el Programa de Apoyo a la Investigación Estudiantil (PAIE) de la CSIC;

- difusión de llamados a actividades de extensión, tanto centrales como de la FIC.

\section{e) Acceso a la información}

- Los docentes mencionan cuatro vías a través de las cuales se informa sobre los distintos aspectos vinculados a la implementación de los planes de estudio (por ejemplo, modificaciones de las mallas curriculares, disposiciones, reglamentos, etcétera):

○ referentes institucionales que incluyen coordinadores de carrera, UAE, coordinador departamento, funcionarios de bedelía;

○ sitio web de la FIC;

- personas concretas que cumplen o han cumplido ciertos roles en la institución (funcionarios TAS, ex coordinadores de carrera);

○ resoluciones del Consejo de la FIC y comunicados enviados desde las coordinaciones de departamento. 


\subsection{Conversatorio}

Luego de una breve presentación de los objetivos del proyecto [5] y sobre los aspectos centrales de los resultados, el conversatorio fue dinamizado a partir de cuatro preguntas clave: ¿se sienten identificados con los resultados compartidos?, ¿qué elementos no mencionados incorporarían a la discusión?, ¿qué acciones se podrían implementar para atender las problemáticas o dificultades encontradas? y, más específicamente, ¿cuáles desde su rol como docentes?

Este espacio de encuentro contó con la participación de 22 docentes: 16 del Instituto de Información y 6 del Instituto de Comunicación, en el cual se dio un intercambio fluido y fructífero en relación con las temáticas surgidas de las preguntas disparadoras.

A continuación, se presentan los aspectos más relevantes, productos del intercambio durante esta instancia.

- Uno de los aspectos principales a tener en cuenta es que existe un interés manifiesto en la temática $\mathrm{y}$, a su vez, se pudo comprobar que los participantes en el conversatorio coinciden, en un gran número, con aquellos docentes que estuvieron presentes en los intercambios realizados durante las reuniones con los distintos departamentos de la Facultad. Los docentes participantes se sintieron, en general, identificados con los resultados de la investigación.

- Tomando en cuenta el intercambio durante el conversatorio, se puede observar que no aparecieron nuevas temáticas emergentes de las surgidas en las reuniones con los departamentos. No obstante, el énfasis en la importancia de continuar con el análisis de la implementación de los PE y, específicamente, en relación con el grado de cumplimiento de los objetivos planteados en los documentos, ha sido un aporte en esta instancia de intercambio.

- Por este motivo, surgió la idea de que se refuerce el vínculo entre el Observatorio de las Profesiones de la Comunicación [6] y el colectivo de egresados de Archivología y Bibliotecología, con la finalidad de establecer un mayor diálogo entre el mundo académico y el profesional con respecto 
a la inserción laboral de los egresados de los PE 2012. Estas acciones conjuntas podrían lograr una retroalimentación con los egresados en cuanto a la reformulación de la implementación de los PE.

- Un aspecto importante para destacar es la dificultad que implica el desarrollo del trabajo final de grado en las trayectorias de los estudiantes. Se consideró que es fundamental trabajar sobre estas dificultades en el marco de cada una de las carreras, así como en la interna de los equipos docentes que llevan adelante la orientación de los mencionados trabajos.

- Como último aspecto a destacar, se considera de suma importancia la continuidad de estos espacios de intercambio y reflexión en el marco del colectivo docente de nuestra facultad para encontrar respuestas en conjunto a las problemáticas comunes.

\section{Conclusiones}

Se observa que los docentes hacen un uso indistinto entre los conceptos plan de estudio, malla curricular e implementación. A su vez, señalan conocer el PE y la mayoría dice considerarlo a la hora de elaborar los programas de sus unidades curriculares. Además, es de hacer notar que, al consultar a los docentes sobre cuándo fue la última vez que tomaron contacto con el documento, no todos los que expresaron conocerlo lo leyeron por última vez en el 2019 o en el 2020.

La mayor parte del cuerpo docente menciona estar al tanto de las disposiciones y de las actualizaciones de la malla curricular. No obstante, gran número de los encuestados no se siente seguro a la hora de responder a las preguntas de los estudiantes sobre algún aspecto del PE. Si bien la mayoría de los docentes dice conocer el PE, se infiere que este documento no es de consulta permanente y no se incluye como rutina informarse sobre su implementación para la orientación de los estudiantes. Esto puede deberse a que los docentes no son identificados especialmente por los estudiantes como una fuente de consulta, por lo que los primeros no sienten la necesidad de consultar al PE ni de informarse en profundidad sobre su implementación. 
La complejidad de la implementación de PE con enfoques pedagógicos flexibles queda de manifiesto en la diferencia promedio entre la cantidad de ingresos y la cantidad de egresos. Aunque habría que afinar los números, es evidente que hay rezago y desvinculación, y otros estudios, que hemos mencionado en los antecedentes, dan cuenta de ello. En relación con lo anterior, poco más de la mitad de los docentes tiene conocimiento sobre la cantidad de egresos y avances estudiantiles. De esto se puede inferir que los docentes no reparan especialmente en las trayectorias estudiantiles.

La facultad cuenta con un equipo de doscientos docentes. No obstante, se observa que solo un grupo reducido de ellos ha participado de las instancias de implementación de este estudio y ha demostrado un elevado interés en la temática abordada. Estos, además, consideran la necesidad de seguir contando con espacios de discusión y debate sobre los PE y su implementación.

Finalmente, consideramos fundamental el rol de los docentes, tanto para la orientación de los estudiantes como en la promoción de sus avances en la carrera. Por lo cual es necesario su involucramiento en los aspectos concernientes a la propuesta pedagógica institucional. Resulta necesario continuar realizando estudios para reunir insumos que permitan reflexionar sobre el tema y que estos promuevan un mayor compromiso de los actores institucionales: docentes, estudiantes, egresados, coordinadores de carrera, directores y órganos de cogobierno.

\section{Referencias bibliográficas}

Díaz Villa, M. (2002). Flexibilidad y Educación Superior en Colombia. Bogotá: ICFES. Recuperado de https://nutricion.fcm.unc.edu.ar/wpcontent/uploads/sites/16/2013/06/FLEXIBILIDAD_Y_EDUCACI_N_SU PERIOR_EN_COLOMBIA.pdf

Ezcurra, A. M. (2007). Los estudiantes de nuevo ingreso: democratización y responsabilidad de las instituciones universitarias. Universidad Nacional de San Pablo. Recuperado de https://www.prg.usp.br/wpcontent/uploads/ana_maria_scurra_caderno_2.pdf

Universidad de la República. (2011). Ordenanza de estudios de grado y otros programas de formación terciaria. Recuperado de 
https://www.cse.udelar.edu.uy/wpcontent/uploads/2013/12/documento_ordenanza_de_grado_corregida_pagi nas_simples.pdf

Universidad de la República. (2012a). Plan de Estudios para las carreras de grado de la EUBCA: Licenciatura en Bibliotecología y Licenciatura en Archivología. Recuperado de https://fic.edu.uy/ensenanza/grado/lic-enbibliotecologia

Universidad de la República. (2012b). Plan de Estudios. Licenciatura en Comunicación. Recuperado de https://fic.edu.uy/ensenanza/grado/lic-encomunicacion

\section{Notas}

[1] Cabe mencionar que el PE de las licenciaturas en Archivología y en Bibliotecología se implementó en el 2012 para las carreras que se ofrecen en el departamento de Paysandú.

[2] Los estudiantes que ingresaron después del 2016 aún están transitando por las carreras.

[3] El Departamento de Metodología es el único que no tiene adscripción en los institutos y es transversal a ellos.

[4] En cuanto a las actividades electivas, los docentes plantean el problema de los horarios y de la insuficiencia de cupos para su cursado.

[5] Se convocó a los docentes dos semanas antes de la fecha del conversatorio y se les compartió un resumen ejecutivo.

[6] El Observatorio de las Profesiones de la Comunicación propone un trabajo activo en dos direcciones interrelacionadas: diálogo crítico con el mundo profesional y revisión de las prácticas y sustentos de la formación universitaria para el ejercicio de las profesiones.

\section{Nota del autor}

Financiamiento: Este estudio contó con la financiación de la Comisión Sectorial de Enseñanza, Udelar, en el marco de la convocatoria a proyectos para el seguimiento y monitoreo de los planes de estudios. 


\section{Notas del editor}

El presente manuscrito fue aprobado para su publicación por Mario Barité

La corrección de estilo del presente texto fue realizada por Katherine Chamyan en el marco del convenio celebrado entre la FHCE (Tecnicatura Universitaria en Corrección de Estilo-Facultad de Humanidades y Ciencias de la Educación) y la FIC (Facultad de Información y Comunicación).

\section{Nota de contribución autoral}

Todos los autores han contribuido en partes iguales para la realización de este artículo. 